\title{
Exact solutions to the Grad-Shafranov equation for magnetically confined plasmas
}

\author{
A. H. Khater and S. M. Moawad \\ Department of Mathematics, Faculty of Science, Beni-Suef University, Beni-Suef, Egypt \\ email: khater_ah@hotmail.com \& smmoawad@hotmail.com
}

\begin{abstract}
A class of exact analytic solutions to the Grad-Shafranov equation (GSE), in cylindrical geometry under assumption of axial symmetry is obtained. For this configuration all the physical quantities are invariant under rotations about a fixed direction, which we take it to be the direction $\mathrm{Oz}$ of a cylindrical polar coordinate system $(r, \theta, z)$. The obtained solutions can be employed to describe isothermal magnetostatic atmosphere.
\end{abstract}

Keywords. Exact solutions, Grad-Shafranov equation, magnetostatic atmosphere

\section{Introduction}

The static equilibrium of the steady state of an axisymmetric ideally conducting plasma is governed by the following partial differential equation (Shafranov (1958); Grad \& Rubin (1958)), written in standard notations and convenient units:

$$
\frac{\partial^{2} \psi}{\partial r^{2}}+\frac{1}{r} \frac{\partial \psi}{\partial r}+\frac{\partial^{2} \psi}{\partial z^{2}}+I \frac{d I}{d \psi}+r^{2} \frac{d P}{d \psi}=0
$$

where $\psi$ is the poloidal magnetic flux, $P=P(\psi)$ is the plasma pressure and $I=I(\psi)=$ $r B_{\theta}$ (where $B_{\theta}$ is the toroidal component of the magnetic field), (see for example Maschke (1972); Woolley (1977); Maschke \& Perrin (1980)). Exact solutions of the GSE are very useful for theoretical studies of plasma equilibrium, transport and magnetohydrodynamic stability. Analytic solutions to the GSE were reported in Zheng, Wootton \& Solano (1996), McCarthy (1999) and Atanasiu, Günter, Lackner \& Miron (2003).

In this article, we present a new exact solution to (1.1) by taking $I=$ const and $P=\alpha+\beta / \psi^{2}$. In this case (1.1) is nonlinear, in which we solve it in section 2 .

\section{Exact solutions}

Using the new variable $x=A r^{2}+\eta(z)$ with $\left(\eta^{\prime}\right)^{2}=4 a \eta+b$, in (1.1), where $A \neq 0, a$ and $b$ are real constants and $\eta$ is a function of $z$, we get

$$
[4 \alpha(x-\eta)+4 a \eta+b] \frac{d^{2} \psi}{d x^{2}}+2 a \frac{d \psi}{d x}-\frac{2 \beta(x-\eta)}{A \psi^{3}}=0 .
$$

Equating to zero the coefficients of like powers of $\eta$ in $(2.1)$, we get

$$
4(a-A) \frac{d^{2} \psi}{d x^{2}}+\frac{2 \beta}{A \psi^{3}}=0, \quad(4 A x+b) \frac{d^{2} \psi}{d x^{2}}+2 a \frac{d \psi}{d x}-\frac{2 \beta x}{A \psi^{3}}=0 .
$$

The solution which satisfies the two equations in $(2.2)$ is $\psi=(c / 2) \sqrt{b+4 a x}$ with $\beta=$ $A a^{2} c^{4}(a-A) / 2$, where $c$ is an integration constant. Therefore the solution of (1.1) is 
(a)

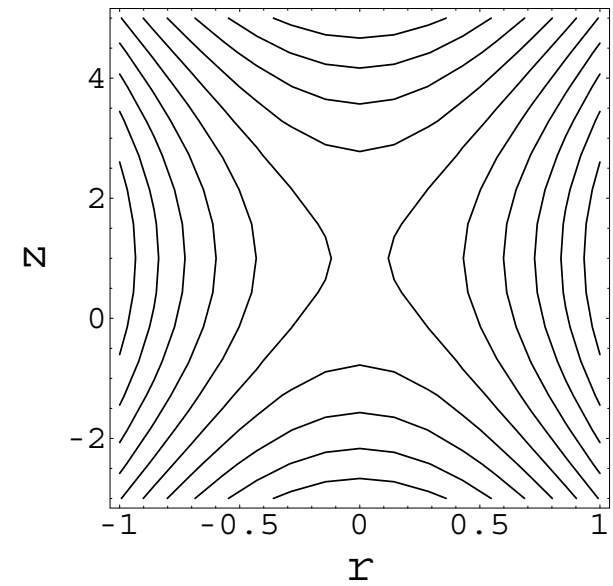

(b)

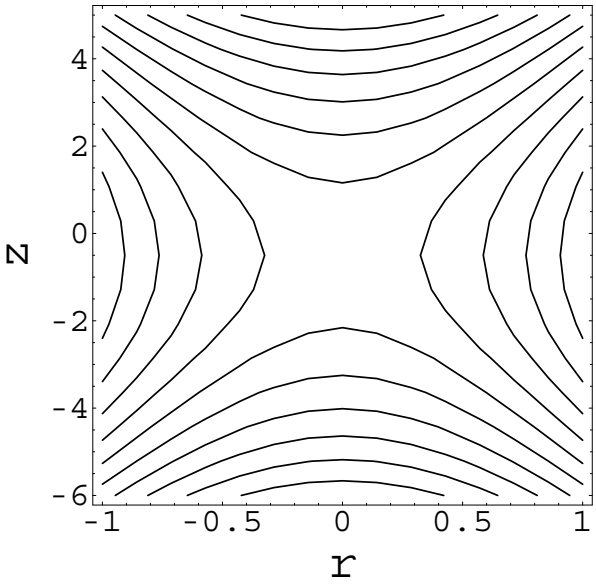

Figure 1. Cross-section of an axisymmetric flux surface for solution (2.3). The values for parameters used are (a) $a=0.5, d=0.5$ and $\alpha=-10$.

$$
\psi=c \sqrt{a A r^{2}+a^{2}(z+d)^{2}},
$$

where $d$ is an integration constant due to solving the equation $\left(\eta^{\prime}\right)^{2}=4 a \eta+b$.

Depending on the value of $d$ the equilibrium configuration can have stagnation points at the positions $(r=0, z=-d)$, where $\psi=0$. Figures 1 (a) and 1(b) shows a crosssection of an axisymmetric flux surface for solution (2.3). The parameter values used in it yield two pairs of open curves about the axes $(r=0, z=-1 / 2)$. In figures 1 (a) and $1(\mathrm{~b})$, the magnetic axis coincide with the axis of symmetry and then the equilibrium becomes a cusp-mirror-like configuration. Solution (2.3) can describe a divertor-tokamaklike configuration with the divertor plate being located close to extremum point of flux.

\section{Summary}

We have obtained a class of exact analytic solutions to nonlinear GSE. This class of solutions has not been reported in literature to our best of knowledge. It may be help in understanding the physics involved in the transition from the low-confinement to the high-confinement mode in tokamaks. Further it can be employed to describe isothermal magnetostatic atmosphere.

\section{References}

Shafranov, V.D. 1958 Sov. Phys. JETP 6, 545

Grad, H. \& Rubin, H. 1958 Proc. Second United Nations Int. Conf. on the Peaceful Uses of Atomic Energy, (United Nations, Geneva), vol. 31, p. 190

Maschke, E.K. 1972 Plasma Phys. 15, 535

Woolley, M.L. 1977 J. Plasma Phys. 18, 537

De Menna, L. 1977 Nuclear Fusion 17, 455

Maschke, E.K. \& Perrin, H. 1980 J. Plasma Phys. 22, 579

Zheng, S.B., Wootton, A.J. \& Solano, E.R. 1996 Phys. Plasmas 3, 1176

McCarthy, P.J. 1999 Phys. Plasmas 6, 3554

Atanasiu, C.V., Günter S., Lackner K. and Miron I.G. 2003 Proceedings of the 30th EPS Conference on Controlled Fusion and Plasma Physics, St. Petersburg, ECA vol. 27A, p-2 104 